\title{
ASSESSMENT OF LOCAL AIR QUALITY FOR DIFFERENT PENETRATION LEVELS OF CONNECTED AUTONOMOUS VEHICLES
}

\author{
LUÍS P. CORREIA ${ }^{1}$, SANDRA RAFAEL ${ }^{1}$, DIOGO LOPES ${ }^{1}$, JORGE BANDEIRA ${ }^{2}$, \\ MARGARIDA C. COELHO ${ }^{2}$, MÁRIO ANDRADE ${ }^{2}, \mathrm{CARLOS} \mathrm{BORREGO}^{1} \&$ ANA I. MIRANDA ${ }^{1}$ \\ ${ }^{1}$ CESAM \& Department of Environment and Planning, University of Aveiro, Portugal \\ ${ }^{2}$ TEMA, Department of Mechanical Engineering, University of Aveiro, Portugal
}

\begin{abstract}
Over the last decade, air quality patterns in Europe have improved due to the implementation of effective policies and actions to reduce atmospheric emissions. Despite of this effort, up to $30 \%$ of European citizens living in cities are still exposed to air pollution levels exceeding European air quality standards. Road transport is one of main emission sources, especially of harmful pollutants such as nitrogen oxide compounds (NOx). Technological developments in recent years have allowed for an increasing level of connected autonomous vehicles (CAVs), restricting human driving behaviour, which provides greater control and standardization of the driving style. The connection of these vehicles to several infrastructures and other road transports makes way to new routing methods that may include, for example, real-time traffic information, improve road safety, commute times and generate environmental benefits. In this context, the main goal of this work was to evaluate the impact of the increasing market penetration of CAVs in local road transport emissions and its influence on air quality. A main avenue of the Aveiro urban area (Portugal) was selected as case study and three scenarios were analysed: i) a baseline case, aiming to characterize the impacts of current car fleet on air quality; ii) one scenario with a $30 \%$ CAVs market penetration rate; and iii) one scenario with a $30 \%$ electric CAVs market penetration rate. The atmospheric emissions were calculated using the vehicle-specific power (VSP) model in an hourly basis for a typical workday. Cars following the parameters of CAVs were changed in order to reduce emissions of a mixed traffic flow composition. To perform the air quality simulations, the Computational Fluid Dynamic (CFD) model VADIS was applied and the NOx concentrations were analysed. Preliminary results indicate that an increased penetration rate of CAVs causes an increase in the NOx emission of the regular and CAVs fleet, and a slight depreciation of the air quality is noticed. However, when considering pure battery CAVs, the NOx concentration at a height of $1.5 \mathrm{~m}$ decreases significantly, averaging a $4 \%$ decrease for the whole computational domain.

Keywords: connected autonomous vehicles, air quality, urban areas, numerical modelling, pollutant dispersion.
\end{abstract}

\section{INTRODUCTION}

In 2016 the road transport sector was the largest contributor to total NOx emissions in EU-28. Despite the European statistics pointed out for an increase of transported passenger, the efforts made in recent years, to apply abatement strategies led to a significant reduction of air pollutants emissions. In fact, recent European air quality reports show that NOx emissions related to the road transport sector have decreased significantly ( $50 \%$ of 2000 levels in 2016) [1]. This trend is extremely important since the contribution of the road transport sector to population exposure to nitrogen dioxide concentrations is high, especially in cities; this occurs since the road traffic emissions are close to the ground and distributed across densely populated areas. As technology evolves at a rapid pace, this sector faces a transformation at a short, medium and long term. At short and medium term, technology makes way for the increase of automation level in the transportation fleet. This allows for a completely new way of increasing road transport energy efficiency and emissions reduction since, until now, the drivers' behaviour has been an uncontrolled vehicle emission factor [2]. 
The real impact of these new measures is definitely related to the market penetration rate of these new vehicles with different automation levels. This direct relation means that for a complete and concise study, the market penetration rate must be considered.

Since the emissions of road transport sector plays a significant role on urban air quality, and its fleet is facing an ever-changing reality, the impacts of these measures should be assessed to ensure that the best path is the one to be chosen. In this context, the main goal of this work is to assess the influence of the inclusion of $30 \%$ of connected autonomous vehicles in an urban fleet on air quality. For that, a main avenue in a medium-sized Portuguese urban area was selected as case study and numerical modelling tools were applied. The paper is structured as followed: Section 2 describes the modelling setup methodology, including a brief description of the case study. The main results are presented in Section 3, including the analysis of road transport emissions and the NOx concentrations. Conclusions follow in Section 4.

\section{METHODOLOGY}

\subsection{Case study description}

For the proposed study, a main avenue called Avenida 25 de Abril of a medium-size city in the Northwest of Portugal, Aveiro, was selected. This avenue is located in the city centre, surrounded by residential buildings and two high schools, contributing to high traffic volume levels at peak hours [3]. Furthermore, this avenue is one of the city's traffic getaways as it connects the city centre to higher flow and interregional roads, and highways. Connections with other roads are scattered throughout the avenue, allowing for a start/stop situation during demanding traffic flow periods. All these features make this avenue a perfect worst-case scenario for CAVs, with plenty parameterization to be made.

The baseline emissions (which characterize the current scenario) were calculated on the traffic emissions model using as inputs the traffic flow characterization, made during an experimental campaign (on 7th February 2017). During this campaign, several factors were studied, including traffic flow speed, time, distance and vehicle counting in an hourly basis.

The wind velocity and direction were measured at height of $10 \mathrm{~m}$, by a meteorological station located in the vicinity of the avenue, for the time interval of the study period. The collected data describes the average hourly wind flow conditions that were used as inflow boundary conditions for the air quality modelling simulations. For the period of the experimental campaign the wind direction was approximately constant from west-northwest (WNW), indicating that the main street canyon is in line with the incoming flow. Significant oscillations in the wind velocity were recorded during the study period, with a maximum variation between 2 and $6 \mathrm{~m} \cdot \mathrm{s}^{-1}$ (Fig. 1(a)).

In addition, an analysis of the meteorological data collected in the same situ in the last five years (2012-2016) was also performed (Fig. 1(b)). According to the data set for the $7^{\text {th }}$ of February 2017, north-westerly quadrant winds blow in this area with a frequency of more than $40 \%$, with the majority of the wind velocity comprised in the range of 2 to $6 \mathrm{~m} \cdot \mathrm{s}^{-1}$ (Fig. 1(a)). Therefore, the study period is representative of the typical meteorological conditions observed over avenue, corresponding also to neutral stability conditions, in agreement with the numerical approach adopted in this study. 


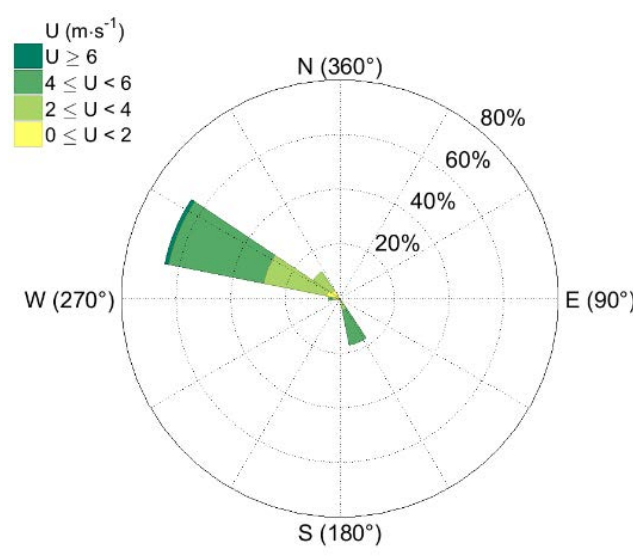

(a)

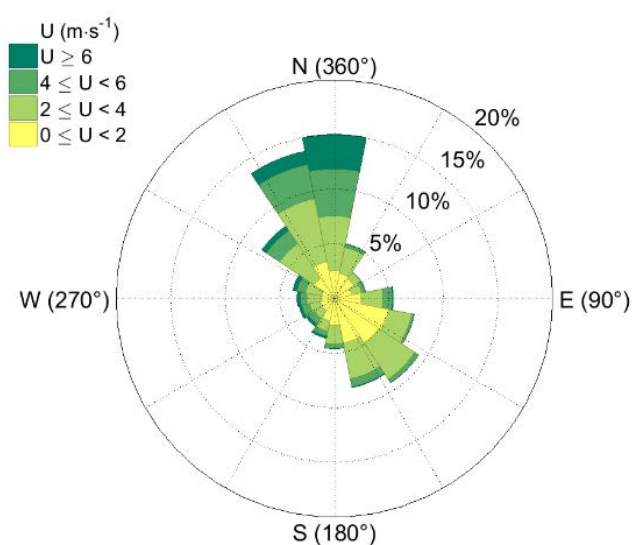

(b)

Figure 1: (a) Wind rose with meteorological data for the 7th of February 2017; and (b) Wind rose with meteorological data for the period between 2012 and 2016.

\subsection{Modelling setup}

\subsubsection{Atmospheric emission approach - VISSIM and VSP}

The impacts of the CAVs operation in the network were assessed using the microscopic traffic model PTV VISSIM. The modelling technique depends on the scope of information available. Ideally, the best approach is to use current commercial driving algorithms, in order to couple the model algorithms. However, given the difficulty in obtaining such data, a method often used to study the CAVs impacts is based on changing the driving parameters of the car following models [4]-[6]. In this study, the parameters optimized by Stogios, in which more than 400 combinations of driving behaviour parameters were evaluated to optimize the environmental performance of vehicles in urban environment, were used. Wiedemann 99 car following model allows for changing 6 many of its parameters and is more suitable for simulating autonomous vehicles. The parameters used are presented on Table 1 . The modelling platform was applied to the study area, with a simulation period of 5400 seconds, in which the first 1800 seconds were considered only for the model to warm up. Then, random seeds were simulated to reflect the stochastic nature of road traffic. The baseline scenario was validated in previous studies [7] including traditional calibration parameters (demand, speed and acceleration data) and also vehicle specific power modal distribution and emissions. Wiedemann car fowling parameters used to simulate CAV's driving behaviour.

Road transport emissions were estimated based on the VSP-based modelling approach. This method provides instantaneous vehicle power per unit of mass. Second by second, VSP values were categorized into 14 bins with a corresponding emission factor for NOx. VSP is a function of speed, acceleration-deceleration and slope [8], calculated using eqn (1),

$$
V S P=v \times[1.1 a+9.81 \sin (\arctan (\text { grade }))+0.132]+0.00302 v^{3},
$$

where $\mathrm{v}$ is the instantaneous speed $\left(\mathrm{m} . \mathrm{s}^{-1}\right)$; $\mathrm{a}$ is the instantaneous acceleration/deceleration $\left(\mathrm{m} . \mathrm{s}^{-2}\right)$, and grade is the road slope (in decimal fraction). 
Table 1: Wiedemann 99 car following parameters.

\begin{tabular}{|l|c|c|}
\hline Parameter & Default behaviour & CAV Stogios 2018 \\
\hline CC0 $(\mathrm{m})$ & 1.50 & 1.47 \\
\hline CC1 $(\mathrm{s})$ & 0.9 & 1 \\
\hline CC2 $(\mathrm{m})$ & 4 & 0 \\
\hline CC3 $(\mathrm{s})$ & -8.00 & -13.54 \\
\hline CC4 $\left(\mathrm{m} . \mathrm{s}^{-1}\right)$ & -0.35 & -0.13 \\
\hline CC5 $\left(\mathrm{m} . \mathrm{s}^{-1}\right)$ & 0.35 & 0.13 \\
\hline CC6 $\left(\left(\mathrm{m} . \mathrm{s}^{-1}\right)\right.$ & 11.44 & 11.44 \\
\hline CC7 $\left(\mathrm{m} . \mathrm{s}^{-2}\right)$ & 0.25 & 0.08 \\
\hline CC8 $\left(\mathrm{m} . \mathrm{s}^{-2}\right)$ & 3.50 & 3.72 \\
\hline CC9 $\left(\mathrm{m} . \mathrm{s}^{-2}\right)$ & 1.50 & 1.60 \\
\hline
\end{tabular}

The microscopic behaviour of traffic, with particular interest in the position of the driver-vehicle unit at $1 \mathrm{~Hz}$ resolution, was the focus of this study. As VSP methodology is able to represent the transient behaviour of vehicle dynamics with high-resolution time, it is an appropriate approach to estimate emissions as result of microscopic changes in driving behaviour. Emission factors for different vehicles types can be found in the literature: Light Duty Gasoline Vehicles (LDGV) [9], Light Duty Diesel Vehicles (LDDV) [10], and Light Commercial Diesel Vehicles (LCDV) [11].

\subsubsection{Air quality modelling - VADIS}

The air quality of the case study was assessed by applying the CFD model VADIS (pollutant DISpersion in the atmosphere under VAriable wind conditions). The model VADIS was developed to assess air pollutants dispersion at local scale, being structured by two main modules: FLOW and DISPER. The FLOW module is the first one to be used, as it calculates the wind field in the computational domain through the numerical solution of the threedimensional (3D) Reynolds average Navier-Stokes equations applying the $\kappa-\varepsilon$ turbulence closure scheme to calculate the wind, turbulent viscosity, pressure and turbulence kinetic energy. In this module, two different grids are used: the wind and the cartographic grids. The information related to the obstacles (buildings and trees) and to the emission sources locations (e.g. roads) and dimensions is defined on the cartographic grid. The wind field is calculated over an Eulerian Cartesian 3D grid, which is overlaid to the cartographic one and rotates according to the wind direction [12]. The grids dimensions and number of cells in each axis must be defined as a compromise between the required resolution, accuracy and the computational demand. The DISPER module follows the first, calculating the dispersion of the air pollutant across the defined domain, applying the Lagrangian approach to the computation of the 3D pollutant concentration field. The Lagrangian approach assumes that the spatial and temporal dispersion of the mass of pollutant emitted is represented by a large number of numerical particles arbitrarily released in the flow. In each time step, each particle displacement is calculated by the sum of a deterministic component obtained from the velocity field, the stochastic component related with the local turbulence translated by the Langevin stochastic theory and the influence of the fluctuation forces, 
represented by the Langevin equation [13]. Initially, the wind field is calculated considering the stationary conditions (FLOW module) and then the model calculated the displacement of these numerical particles over the cartographic grid (DISPER module). Integrated in the DISPER module, the Urban Vegetation (URVE) module, accounts for the aerodynamic effects of trees over the 3D wind field. Consequently, the dispersion of the emitted air pollutants is conditioned by vegetation through this disturbed wind flow, allowing a better representation of the flow and dispersion of air pollutants in urban environments. The magnitude of this perturbation depends most of all on the characteristics of the vegetation itself (e.g., location, size) and of the incoming airflow (e.g., velocity, direction, turbulence). The output provided by VADIS includes the three wind velocity components, the turbulent viscosity, the turbulent kinetic energy, the turbulent dissipation and the pollutant concentration in each grid cell for the entire cartographic grid.

VADIS performance has been improved through the years, based on the comparison of modelled data with wind tunnel measurements and data from air quality monitoring stations [12], [14]-[17]. Currently, VADIS has the capability to support multi-obstacle (e.g. buildings and trees) and multi-source description as well as, time varying flow fields and time varying emissions, allowing the evaluation of maximum short-term local concentrations in urban geometries [17].

To define the computational domain (Fig. 2), the guidelines proposed by COST Action 732 [18] were followed. For the simulation of urban flows with multiple buildings, the extension of each direction of the domain were set with a minimum of $5 \mathrm{H}_{\max }$, where $\mathrm{H}_{\max }$ represents the height of the tallest building. This way, the domain volume was $958 \times 958 \times 86 \mathrm{~m}^{3}(\mathrm{~L} \times \mathrm{W} \times \mathrm{H})$ and mesh resolution of $2 \times 2 \times 2 \mathrm{~m}^{3}$ was used. The obstacles in the domain were of two types: buildings and trees. These were characterized according to a geographic information system (GIS), which allows the creation of a virtual configuration of the study area. The obstacles are then simplified by assembling adjacent volumes with similar characteristics. The complex shape of trees was also simplified, defined as parallelepipeds positioned at a given distance above ground, corresponding to the average trunk height. The generation of the urban objects (3D buildings, trees and roads) have been virtually defined in VADIS using the geometry pre-processors developed, based on the coordinates of the objects. The case study complies a total number of 192 buildings and 241 trees.

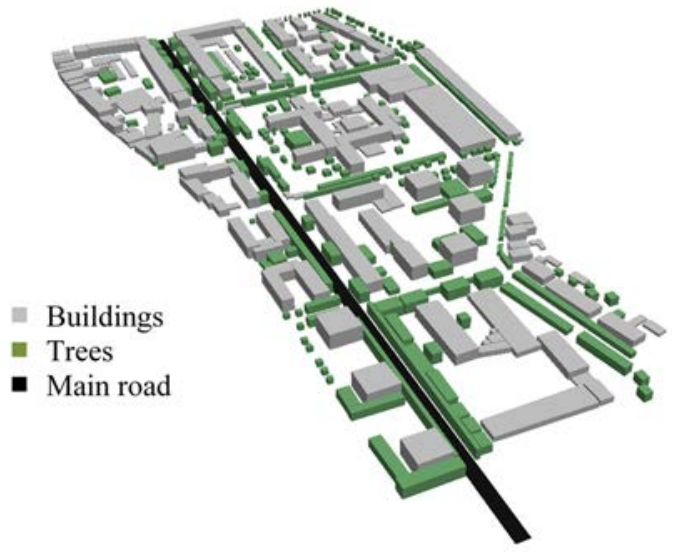

Figure 2: Computational domain used for air quality modelling. 
The numerical simulations were carried out for NOx in an hourly basis, for a workday period.

\subsection{Autonomous vehicles scenarios}

In 2016, the European Commission wrote a roadmap to autonomous and connected driving in 2030. In this report, it is stated that autonomous driving in urban areas will be possible in some cities, already between 2024 and 2030 [19]. However, the penetration rate of electric vehicles is still uncertain. In this study, two extreme cases to make a sensitivity analysis of the impact of this factor were selected: scenario 1) where a $30 \%$ penetration rate of CAVs was considered, assuming an engine technology in line with the remaining fleet; and scenario 2) with the same $30 \%$ penetration rate of CAVs, accounting however that this fraction of vehicles are pure battery electric vehicles (BEV).

Results from each scenario, acquired from the emission modelling, where then applied to the baseline emission results. For scenario 1, a total fleet, overall increase of $1.60 \%$ in the NOx emission was observed. This increase occurred due to the slight increase $(1.6 \%)$ of traffic demand caused by the better traffic flow performance resulting from the use of CAVs in the traffic fleet. For scenario 2, it was considered that the NOx emission of $30 \%$ of the fleet was zero, bearing in mind that this fraction of the fleet consists of pure battery electric CAVs. Nevertheless, the remaining fraction of the fleet was still affected by the introduction of CAVs in the network, resulting in a NOx emission increase of $1 \%$. However, the total fleet emission rate still decreases $29.3 \%$ on average. Fig. 3 shows the daily profile, in an hourly basis, of the NOx emission rate in the avenue for baseline and both scenarios.

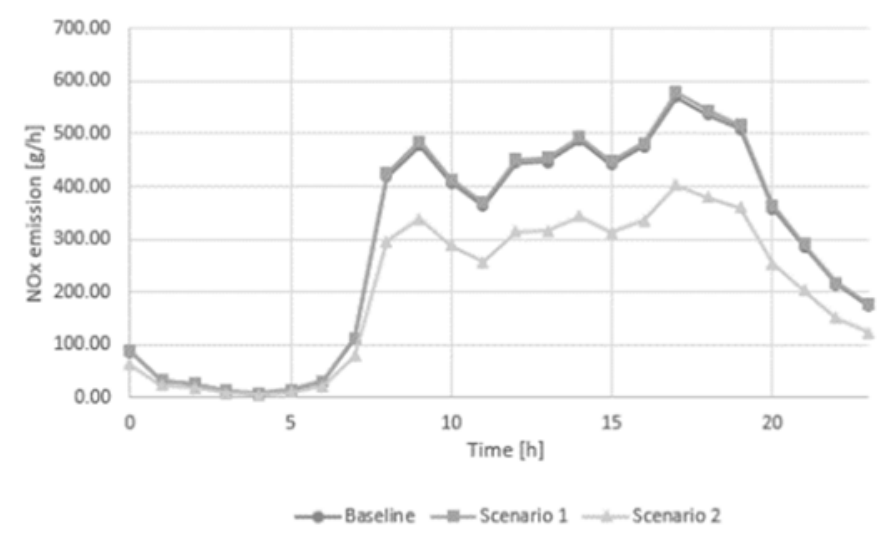

Figure 3: Daily profile at an hourly basis of the NOx emission rate.

\section{RESULTS AND DISCUSSION}

\subsection{Air quality}

On all scenarios, the air quality was evaluated according to the hourly concentration of NOx at a height of $1.5 \mathrm{~m}$. The maximum value of NOx concentration (spatial average) occurred on the same time period for all scenarios (between 9 and 10 a.m.), with a value of $10.61 \mu \mathrm{g} . \mathrm{m}^{-3}$ for the scenario 1 , followed by $10.37 \mu \mathrm{g} . \mathrm{m}^{-3}$ for baseline and $7.36 \mu \mathrm{g} \cdot \mathrm{m}^{-3}$ for 
scenario 2. On the baseline case, NOx concentration is lowest and near zero between midnight and 7 a.m., time at which it rises to $10 \mu \mathrm{g} . \mathrm{m}^{-3}$ until $10 \mathrm{a} . \mathrm{m}$., when it lowers to a concentration value of $3.40 \mu \mathrm{g} \cdot \mathrm{m}^{-3}$ (spatial average). From there, the concentration keeps relatively constant until another peak arises between 5 and 7 p.m. with a value of about $6 \mu \mathrm{g} . \mathrm{m}^{-3}$, decreasing to $4.42 \mu \mathrm{g} . \mathrm{m}^{-3}$ at $8 \mathrm{p} . \mathrm{m}$., stabilising until $11 \mathrm{p} . \mathrm{m}$. For both scenarios this behaviour is held, with a difference in magnitude of the value.

Fig. 4 shows the percentual hourly difference of NOx concentrations (average of domain) of the analysed scenarios compared with the baseline. For scenario 1, despite the increase of $1.60 \%$ in the NOx emission, the resulting NOx concentration variation increase is slightly lower, equal to approximately $1 \%$ during peak hours. However, for scenario 2 , the decrease of NOx emission (approximately 29\%) has a significant expression in air quality, reaching to a reduction of $8 \%$ in the NOx concentration variation (comparing to baseline) in the period between 9 and 10 a.m. The reduction of the NOx concentration across the day is very noticeable with a mean value of approximately $4 \%$.

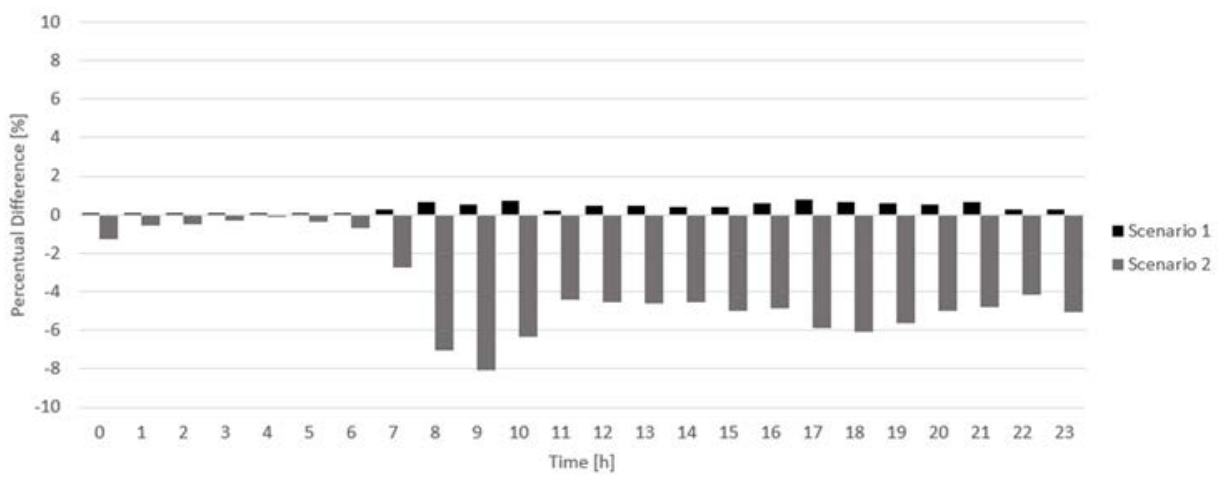

Figure 4: Percentual hourly difference of NOx mean concentration of each scenario in comparison to baseline.

The highest emission rate throw-out in the day occurs at 5 p.m. However, in the air quality analysis, this period of the day is not the one with the worst NOx concentration. This result shows that meteorological conditions have a great influence in the air pollution dispersion over the study area, showing the importance of a dispersion.

As example, the spatial distribution of the percentual difference between scenario 2 and baseline for the period of 9 to 10 a.m. (peak hour) is shown in Fig. 5. The area most affected by the reduction of NOx concentration is located at downwind area, in the vicinity of the road that include residential buildings and both high schools. This result is of great interest since it should positively affect the local population, reducing their exposure to the NOx concentrations, especially important in a residential area, which includes two schools.

\section{CONCLUSIONS}

The air pollution levels of a main avenue of the city of Aveiro was studied, using a street level resolution model, for a typical weekday. The modelling approach consisted of: i) a road traffic flow simulation using driving parameters of CAVs and study their influence in speed and acceleration parameters; ii) the calculation of the atmospheric emissions on an hourly basis through a VSP approach; and iii) the calculation of the NOx concentration applying the CFD model VADIS. 


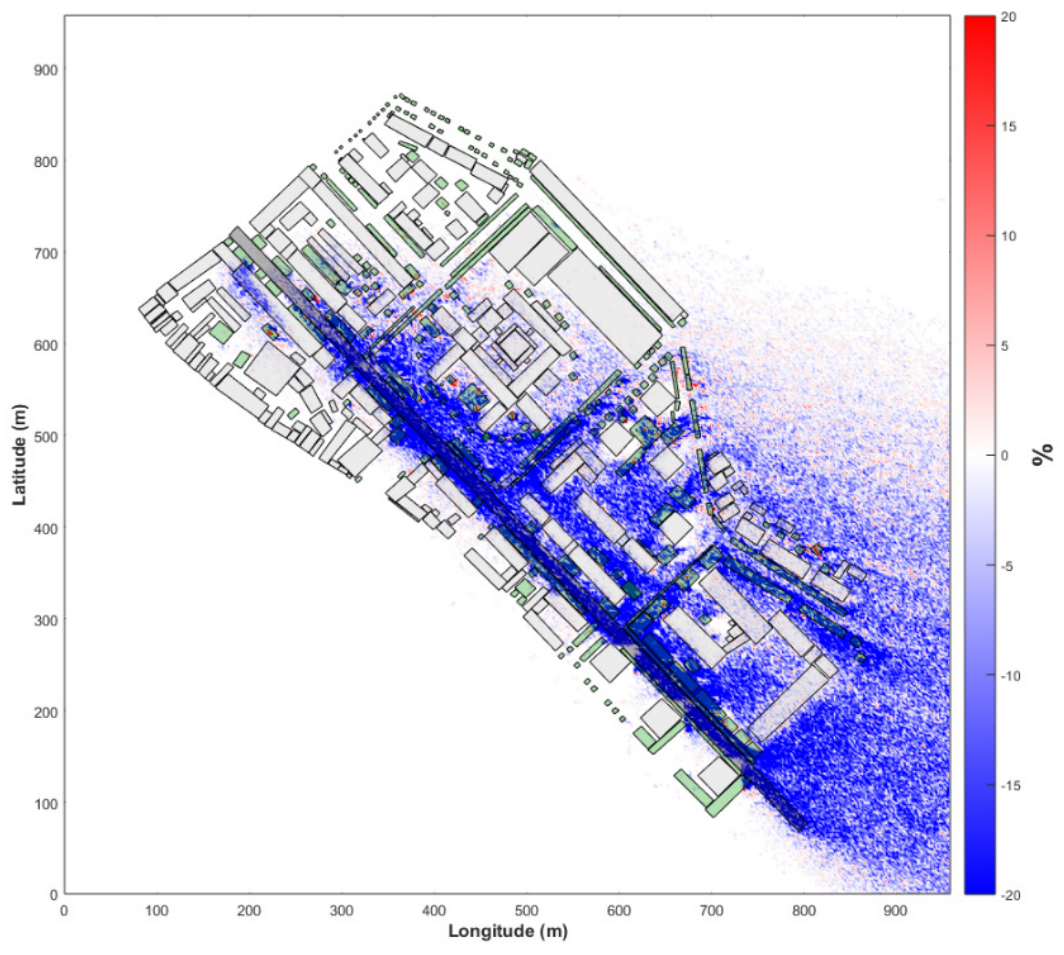

Figure 5: Spatial distribution of the percentual difference between baseline and scenario 2 across the computational domain, for the period between 9 and 10 a.m.

Results show that the introduction of CAVs in the current car fleet can provide both advantages and disadvantages if the car following parameters tested in this study are used. With a $30 \%$ penetration rate of engine technology CAVs, NOx emissions slightly increase for both regular and connected vehicles, resulting in the increase of the NOx concentrations in the surrounding area of the avenue. However, when considering that these CAVs are pure battery vehicles, the air quality around the avenue improved significantly, by a decrease of NOx concentration, despite the slight increase of NOx emission of the remaining $70 \%$ of regular vehicles in the car fleet.

This study shows the importance of numerical models as tools to support decision makers in the adoption of the best urban mobility strategies.

\section{ACKNOWLEDGEMENTS}

The authors acknowledge the support of InFLOWence project (POCI-01-0145-FEDER029679), funded by the Portuguese Science and Technology Foundation through national funds and by the European Community Fund FEDER within the COMPETE2020 program. Thanks are due for the financial support to CESAM (UID/AMB/50017/2019), to TEMA: "Centre for Mechanical Technology and Automation Strategical Project UID/EMS/00481/2013-41 FCT and CENTRO-01-0145-FEDER-022083”, to FCT/MCTES through national funds, and the co-funding by the FEDER, within the PT2020 Partnership Agreement and Compete 2020. 


\section{REFERENCES}

[1] European Environment Agency, Air quality in Europe, report no. 12., 2018.

[2] Nieuwenhuijsen, J., de A. Correia, G. H., Milakis, D., van Arem, B. \& van Daalen, E., Towards a quantitative method to analyze the long-term innovation diffusion of automated vehicles technology using system dynamics. Transportation Research Part C: Emerging Technologies, 86, pp. 300-327, 2018.

[3] Vicente, B. et al., Influence of different complexity levels of road traffic models on air quality modelling at street scale. Air Quality, Atmosphere \& Health, 11(10), pp. 12171232, 2018.

[4] Stogios, C., Investigating the effects of automated vehicle driving operations on road emissions and traffic performance, MASc thesis, University of Toronto, 2018.

[5] Zeidler, V., Buck, S., Kautzsch, L., Vortisch, P. \& Weyland, C., Simulation of Autonomous Vehicles Based on Wiedemann's Car Following Model in PTV Vissim. in Transport Research Board, TRB annual meeting online, 2019.

[6] Lee, S., Jeong, E., Oh, M. \& Oh, C., Driving aggressiveness management policy to enhance the performance of mixed traffic conditions in automated driving environments. Transportation Research Part A: Policy Practice, 121, pp. 136-146, 2019.

[7] Bandeira, J. M., Fernandes, P., Fontes, T., Pereira, S.R., Khattak, A.J. \& Coelho, M.C., Exploring multiple eco-routing guidance strategies in a commuting corridor. International Journal of Sustainable Transportation, 12(1), pp. 53-65, 2018.

[8] US Environmental Protection Agency, User's Guide to Mobile6, Mobile Source Emission Factor Model, 2002.

[9] Anya, A.R. \& Frey, H.C., Method and Case Study for Quantifying Local Emissions Impacts of a Transportation Improvement Project Involving Road Re-Alignment and Conversion to a Multi-Lane Roundabout INTRODUCTION AND MOTIVATION, Transp. Res. Board, 92nd Annu. Meet., 2013.

[10] Coelho, M.C., Farias, T.L. \& Rouphail, N.M., A Numerical Tool for Estimating Pollutant Emissions and Vehicles Performance in Traffic Interruptions on Urban Corridors. International Journal of Sustainable Transportation, 3(4), pp. 246-262, 2009.

[11] Coelho, M.C., Frey, H.C., Rouphail, N.M., Zhai, H. \& Pelkmans, L., Assessing methods for comparing emissions from gasoline and diesel light-duty vehicles based on microscale measurements. Transportation Research Part D: Transport and Environment, 14(2), pp. 91-99, 2009.

[12] Rafael, S., Vicente, B., Miranda, A.I., Borrego, C., Rodrigues, V. \& Lopes, M., Impacts of green infrastructures on aerodynamic flow and air quality in Porto's urban area. Atmospheric Environment, 190, pp. 317-330, 2018.

[13] Lee, R.L. \& Näslund, E., Lagrangian stochastic particle model simulations of turbulent dispersion around buildings. Atmospheric Environment, 32(4), pp. 665-672, 1998.

[14] Borrego, C., Tchepel, O., Costa, A.M., Amorim, J.H. \& Miranda, A.I., Emission and dispersion modelling of Lisbon air quality at local scale. Atmospheric Environment, 37(37), pp. 5197-5205, 2003.

[15] Salmin, L., Amorim, J.H., Janko, J., Tchepel, O., Costa, A.M. \& Borrego, C., Integrated modeling of road traffic emissions: Application to lisbon air quality management. Cybernetics and Systems, 35(5-6), pp. 535-548, 2004.

[16] Richards, K., Schatzmann, M. \& Leitl, B., Wind tunnel experiments modelling the thermal effects within the vicinity of a single block building with leeward wall heating. Journal of Wind Engineering and Industrial Aerodynamics, 94(8), pp. 621-636, 2006. 
[17] Tavares, R., Amorim, J.H., Valente, J., Borrego, C. \& Rodrigues, V., CFD modelling of the aerodynamic effect of trees on urban air pollution dispersion. Science of the Total Environment, 461-462, pp. 541-551, 2013.

[18] Franke, B., Hellsten, J., Schlünzen, A. \& Carissimo, H., Best practice guidelines for the CFD simualtion of flows in the urban environment, COST, Quality assurance and improvement of microscale meteorological models, 2007.

[19] European Commission, Roadmap on Highly Automated vehicles, GEAR 2030 DISCUSSION PAPER, 2016. 